\title{
Endodontic Management of the Maxillary First Molar with Two Palatal Canals: A Case Report
}

\author{
Akanksha Jharwal ${ }^{1}$, Deepak Raisingani ${ }^{2}$, Prachi Mital $^{3}$, Lalita Poonia ${ }^{4}$
}

\section{Abstract}

The major goals of root canal treatment are to remove irritants from the root canal system, fill or obturate the cleaned and shaped system, and prevent future recontamination of sealed root canals. However, endodontic treatment is also dependent on having sound knowledge of the internal anatomy of human teeth, especially when anatomic variations are present, clinicians should carefully explore the pulpal floor, further by changing the X-ray projection angle to confirm the existence of variation and prevent the missed canals, all of which are necessary for a successful root canal therapy. These clinical case reports present the application of cone-beam computed tomography as a useful imaging technique in endodontics for the management of teeth with aberrant anatomy.

Keywords: Anatomic variations, Canals, Cone-beam computed tomography, Maxillary molar, Root canal treatment.

Journal of Mahatma Gandhi University of Medical Sciences \& Technology (2020): 10.5005/jp-journals-10057-0125
\end{abstract}

\section{INTRODUCTION}

Knowledge of internal dental morphology is an extremely important factor in planning and administering root canal therapy. The success of endodontic therapy depends on proper access preparation, thorough shaping and disinfection, and threedimensional leakproof obturation of the entire root canal system. Failure in detecting extracanals and not negotiating the curvatures are prime reasons for treatment failures in endodontics, especially in molars. ${ }^{1}$

The incidence of a maxillary first molar with two separate canals in the palatal root is $<1 \%$. $^{2}$ For successful endodontic treatment in maxillary first molars, the dentist must be prepared for unusual root canal anatomy, and efforts must be directed to understanding these variations. ${ }^{3}$

Filho et al. ${ }^{3}$ assessed the internal morphology of maxillary first molars by three different methods and found that the prevalence of the existence of the second palatal canal as follows: ex vivo assessment-2.05\%, in clinical assessment- $0.65 \%$, and by conebeam computed tomography (CBCT) $-4.55 \%$. They proposed a classification (Fig. 1) based on the root separation level and divergence as follows:

- Type I molars - two widely divergent, long, tortuous palatal roots, buccal roots-cow horn-shaped, less divergent.

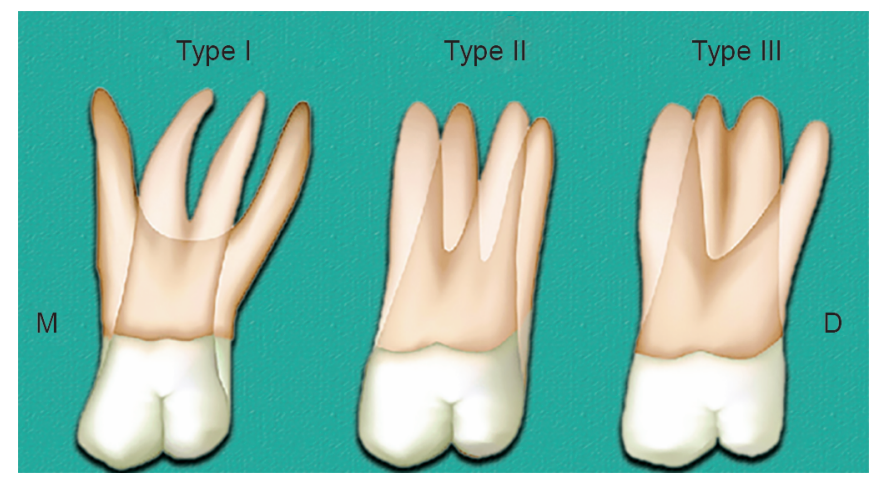

Fig. 1: Classification of four-rooted maxillary first molar ${ }^{3}$

\begin{abstract}
${ }^{1-4}$ Department of Conservative Dentistry and Endodontics, Mahatma Gandhi Dental College and Hospital, Jaipur, Rajasthan, India

Corresponding Author: Lalita Poonia, Department of Conservative Dentistry and Endodontics, Mahatma Gandhi Dental College and Hospital, Jaipur, Rajasthan, India, Phone: +91 9571350505, e-mail: lalitapoonia000@gmail.com

How to cite this article: Jharwal A, Raisingani D, Mital P, et al. Endodontic Management of the Maxillary First Molar with Two Palatal Canals: A Case Report. J Mahatma Gandhi Univ Med Sci Tech 2020;5(2):61-65.

Source of support: Nil

Conflict of interest: None
\end{abstract}

- Type II molars-four separate roots shorter, run parallel, have buccal and lingual morphology, blunt tooth apices.

- Type III molars-mesiobuccal, distobuccal, and palatal engaged in a web of dentin. Distobuccal root may diverge into distobuccal, standing alone.

The present case report highlights the endodontic management of maxillary first molar with the unusual morphology of three roots and four root canals-two palatal, one mesiobuccal, and one distobuccal canal.

\section{Case Descriptions}

\section{Case 1}

A 27-year-old male patient reported at the Department of Conservative Dentistry and Endodontics with the chief complaint of tooth decay in the upper right back tooth region. His medical history was not contributory. Clinically, the tooth had a deep carious lesion on the mesiocclusal surface. On clinical examination, maxillary right first molar (\#16) revealed deep mesioproximal caries which was nontender to percussion. Further evaluation with thermal and electric pulp sensitivity test revealed no response. The initial periapical radiograph revealed a wide palatal root with a fast break-in canal (Fig. 2). Cone-beam computed tomography analysis was done to confirm the root anatomy. Cone-beam computed tomography

(c) The Author(s). 2020 Open Access This article is distributed under the terms of the Creative Commons Attribution 4.0 International License (https:// creativecommons.org/licenses/by-nc/4.0/), which permits unrestricted use, distribution, and non-commercial reproduction in any medium, provided you give appropriate credit to the original author(s) and the source, provide a link to the Creative Commons license, and indicate if changes were made. The Creative Commons Public Domain Dedication waiver (http://creativecommons.org/publicdomain/zero/1.0/) applies to the data made available in this article, unless otherwise stated. 


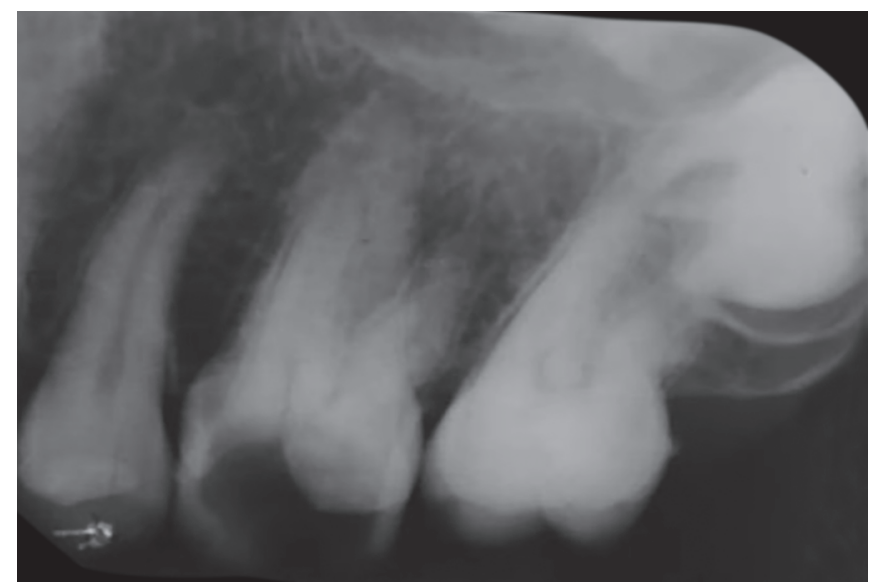

Fig. 2: Preoperative IOPA

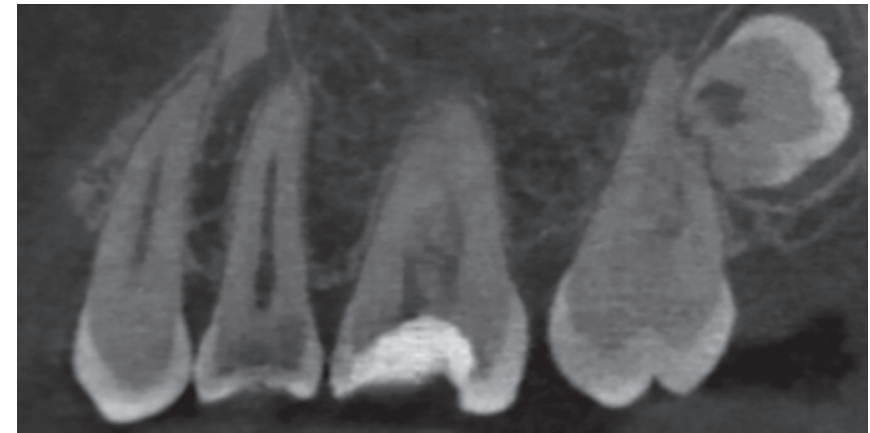

Fig. 4: Sagittal section cone-beam computed tomography

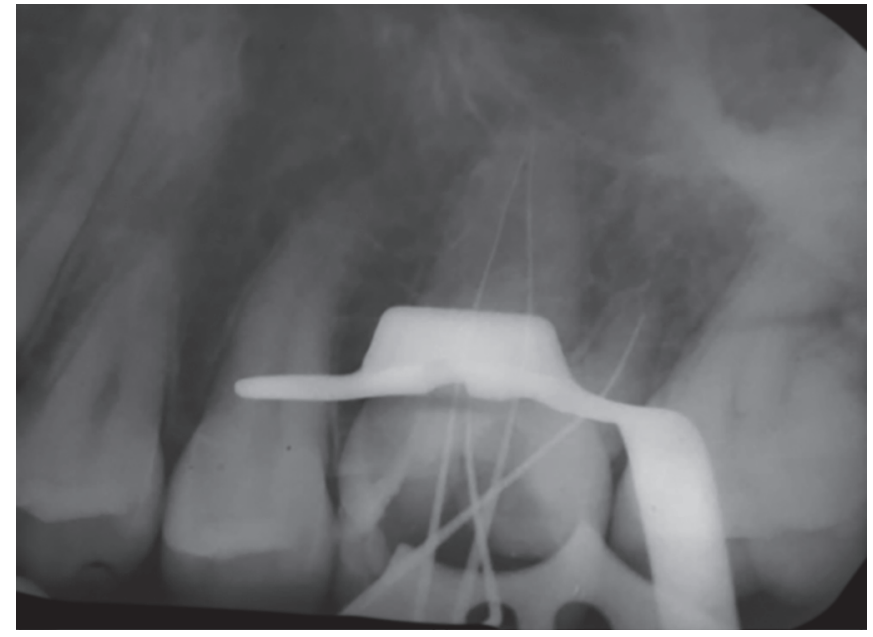

Fig. 6: Working length

revealed the presence of three roots including one palatal root with two canals and two buccal roots (Figs 3 and 4). These findings led to a diagnosis of pulp necrosis with asymptomatic apical periodontitis of the right maxillary first molar, necessitating endodontic therapy.

The patient received local anesthesia of $2 \%$ lidocaine with 1: 80,000 epinephrine. After removing caries of the tooth, a conventional endodontic access opening was made with Endoaccess bur no. 2 (Dentsply Maillefer) (Fig. 5). Clinical examination with a DG-16 endodontic explorer (HuFriedy, Chicago, IL, USA) revealed four distinct orifices: two palatals with two buccal orifices.

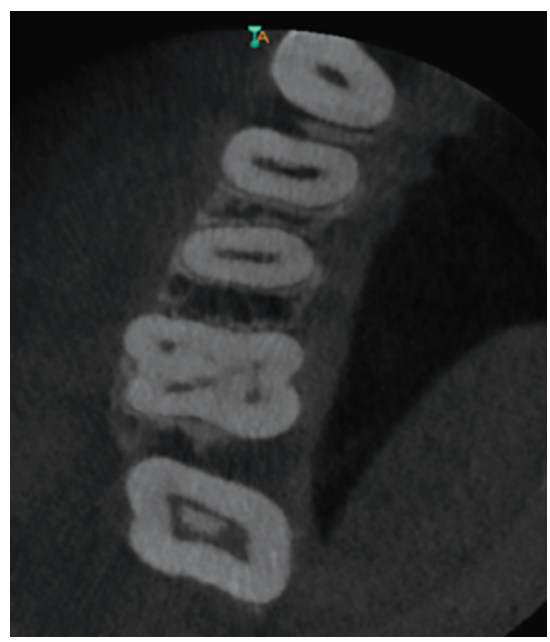

Fig. 3: Axial section cone-beam computed tomography

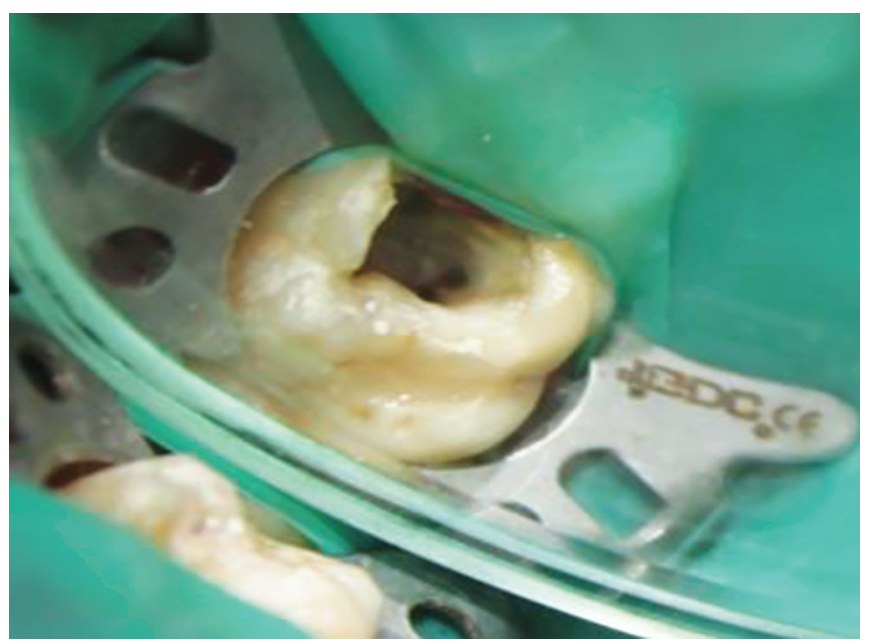

Fig. 5: Access opening

Working length was established with 10K file (Dentsply Maillefer, Switzerland) using apex locator Root ZX (J.Morita, USA) and was confirmed radiographically (Fig. 6). Biomechanical preparation was done by $25-4 \%$ in mesiobuccal and distobuccal canals and $20-6 \%$ in palatal canals by Hyflex CM rotary files (ColtenelWhaledent) using 15\% EDTA (Glyde, Dentsply Maillefer) with thorough irrigation by $5.25 \%$ of sodium hypochlorite and copious saline.

The canals were dried using sterilized paper points (Dentsply Maillefer, Ballaigues, Switzerland) and filled with calcium hydroxide paste (Ivoclar viva dent ApexCal) as an intracanal medicament, and closed dressing was placed (Cavit G, 3M ESPE, Germany).

One week later, the patient was recalled. The patient was asymptomatic, and the medicament was removed with the help of saline irrigation, the root canals were dried using paper point. The master cone fit was determined (Fig. 7). Root canals were obturated with gutta-percha (25-4 and 20-6\%) along with AH Plus resin-based root canal sealer (Dentsply Maillefer) (Fig. 8) followed by composite (filtek Z350 XT, 3M ESPE, Germany) as post root canal filling material and coronal preparation was done and the impression was taken. After 2 days, a porcelain fused metal (PFM) coronal prosthesis was delivered (Figs 9 and 10). 


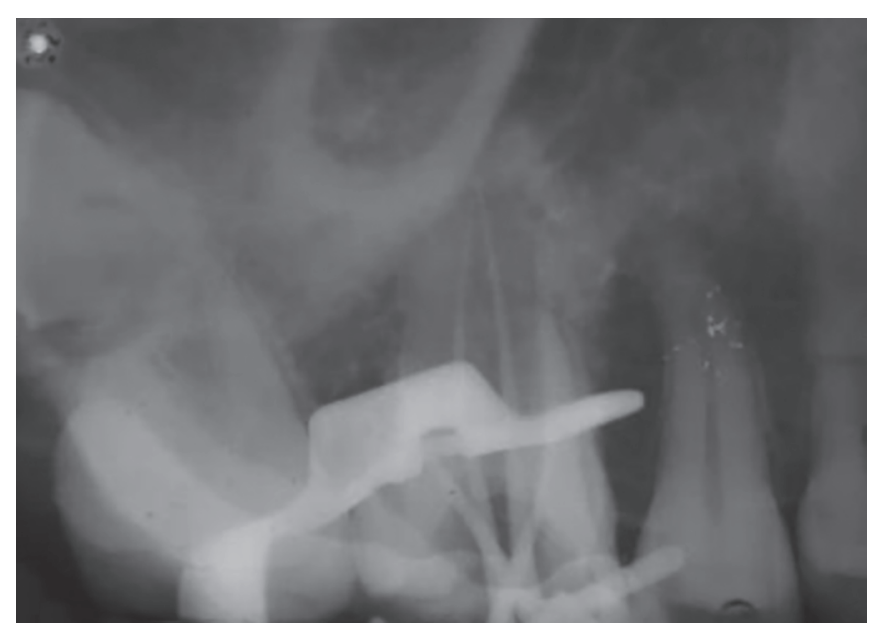

Fig. 7: Master cone

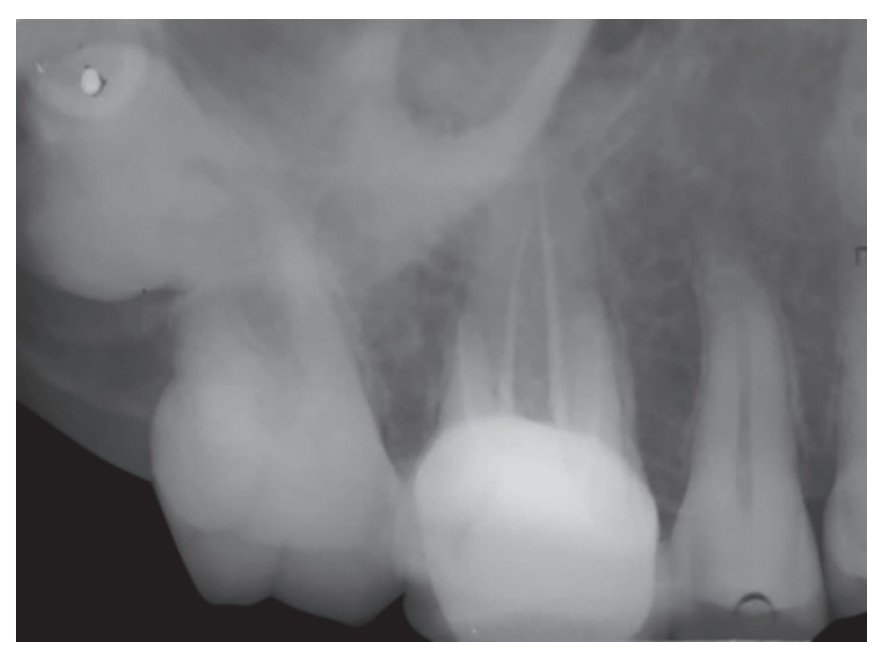

Fig. 9: After coronal prosthesis

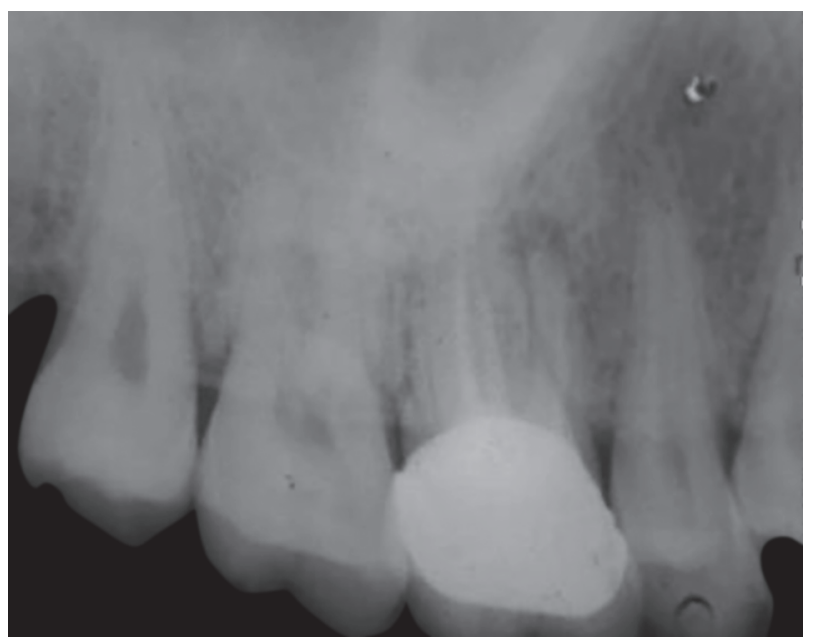

Fig. 11: Preoperative IOPA

\section{Case 2}

A 36-year-old male patient reported at the Department of Conservative Dentistry and Endodontics with the chief complaint of pain and swelling in the upper right back tooth region for 2

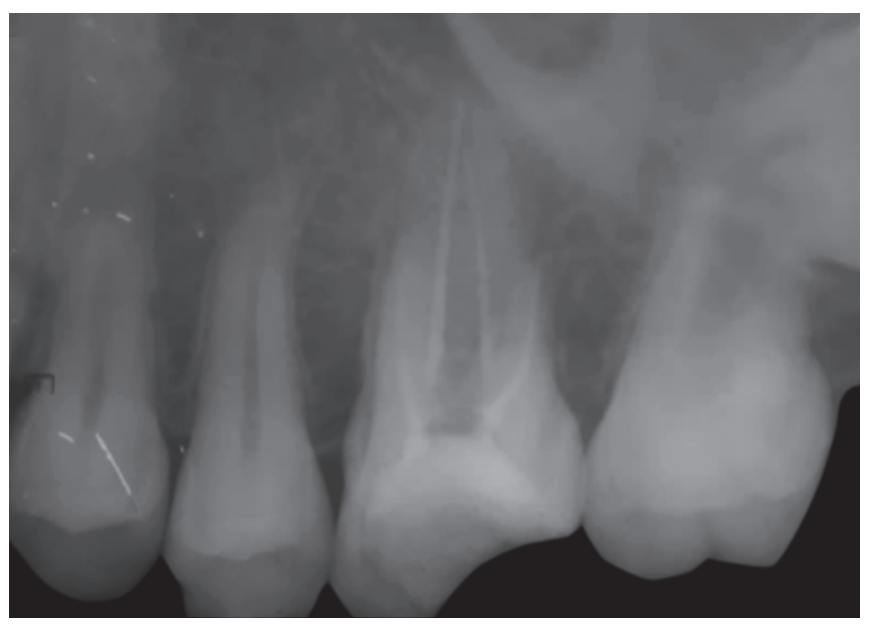

Fig. 8: Obturation

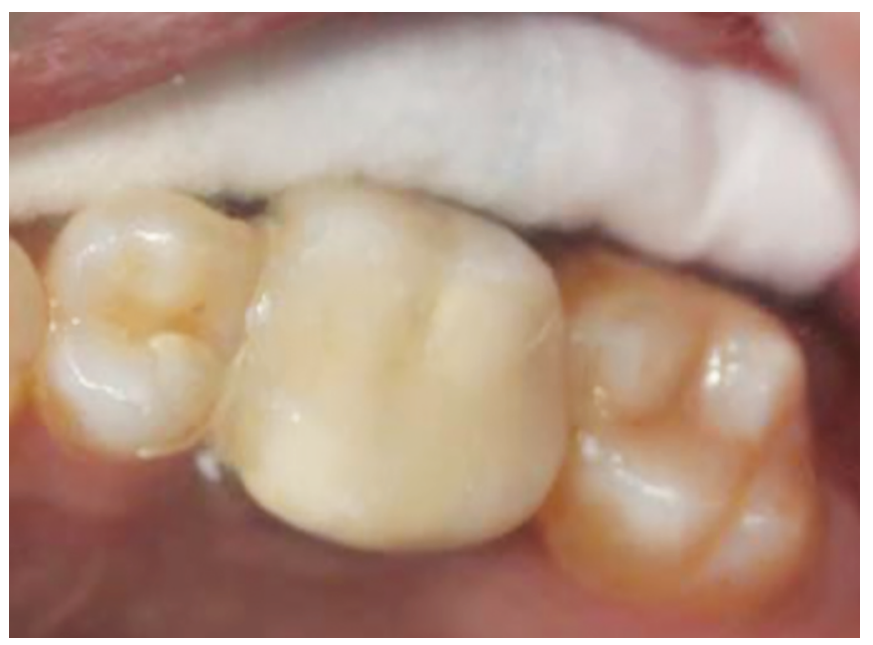

Fig. 10: After coronal prosthesis

days, patient underwent root canal treatment in the same tooth 1 year ago from somewhere out. The patient was not educated enough about the treatment done in the tooth (Fig. 11), his medical history was not contributory. The tooth was tender and swelling was observed associated with \#16. These clinical findings led to the diagnosis of a previously root canal treated with acute periapical abscess. The prosthesis was removed and gutta-percha was retrieved, only $2 \mathrm{~mm}$ of obturated material from the coronal part was removed using no. 2 Gates Glidden drill, gutta-percha was softened with a drop of EndoSolv (Septodont), and retrieval was done with $\mathrm{H}$ files (Fig. 12). Clinical examination with a DG-16 endodontic explorer (Hu-Friedy, Chicago, IL, USA) revealed four distinct orifices: two palatals with two buccal orifices.

Endodontic access opening was modified with Endo $z$ bur (Dentsply Maillefer) (Fig. 13). Clinical examination with a DG-16 endodontic explorer (Hu-Friedy, Chicago, IL, USA) revealed four distinct orifices: two palatals with two buccal orifices.

Working length was established with $10 \mathrm{~K}$ file (Dentsply Maillefer, Switzerland) (Fig. 14) and cleaning and shaping were done till $25-4 \%$ in mesiobuccal and distobuccal canals and $20-6 \%$ in palatal canals by Hyflex CM rotary files (Coltene/Whaledent) using $15 \%$ EDTA (Glyde, Dentsply Maillefer) with thorough irrigation by $5.25 \%$ sodium hypochlorite and copious saline. Chlorhexidine 


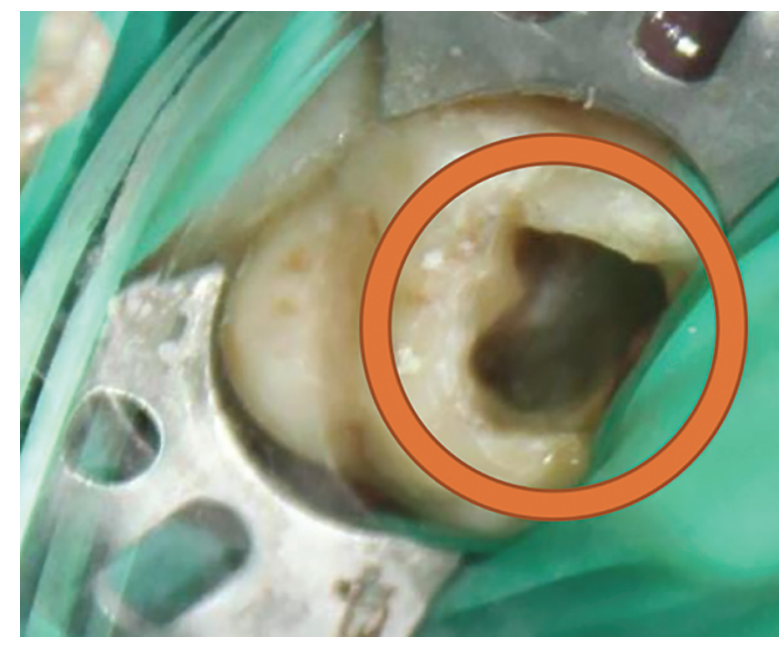

Fig. 12: GP retrieved

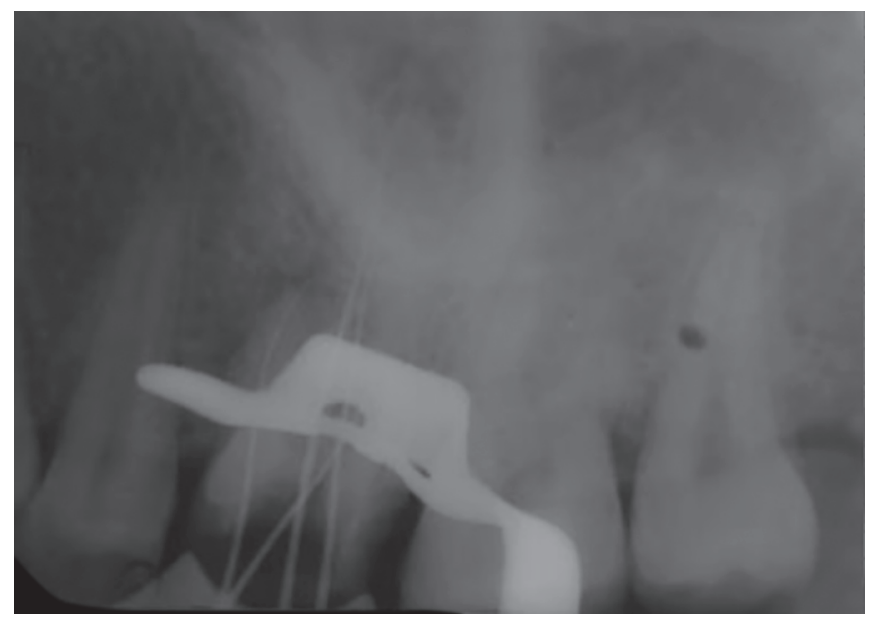

Fig. 14: Working length

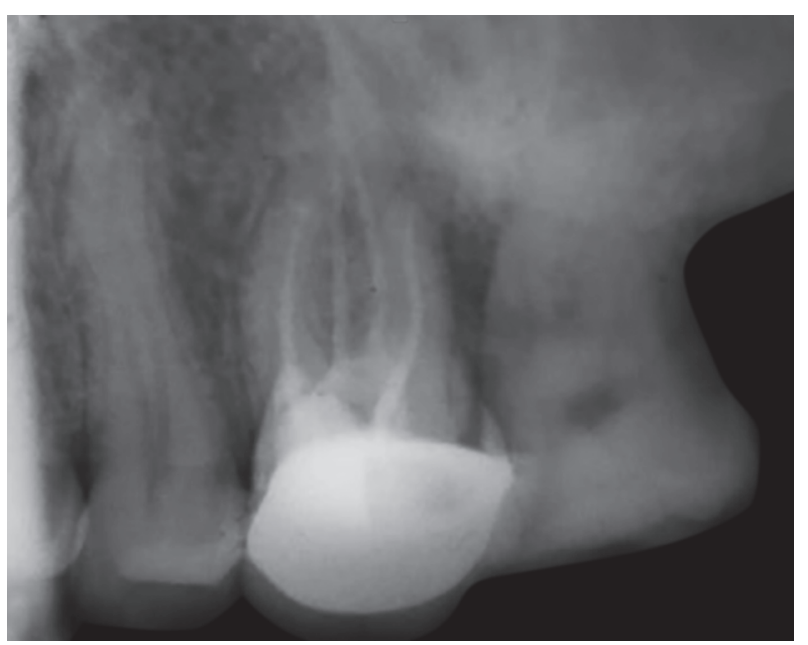

Fig. 16: Obturation

(Dentachlor 2\%) was used as the final rinse. The canals were dried using sterilized paper points (Dentsply Maillefer, Ballaigues, Switzerland) and filled with calcium hydroxide paste (Ivoclar viva

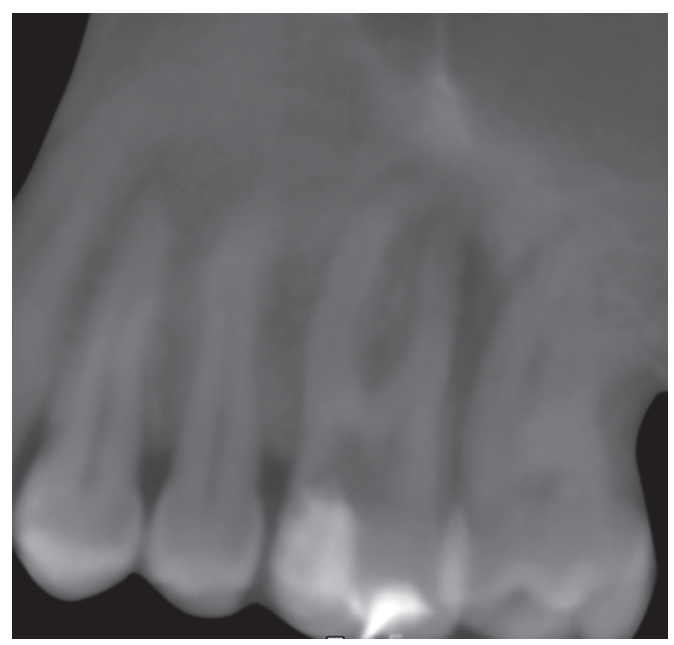

Fig. 13: Modified access opening

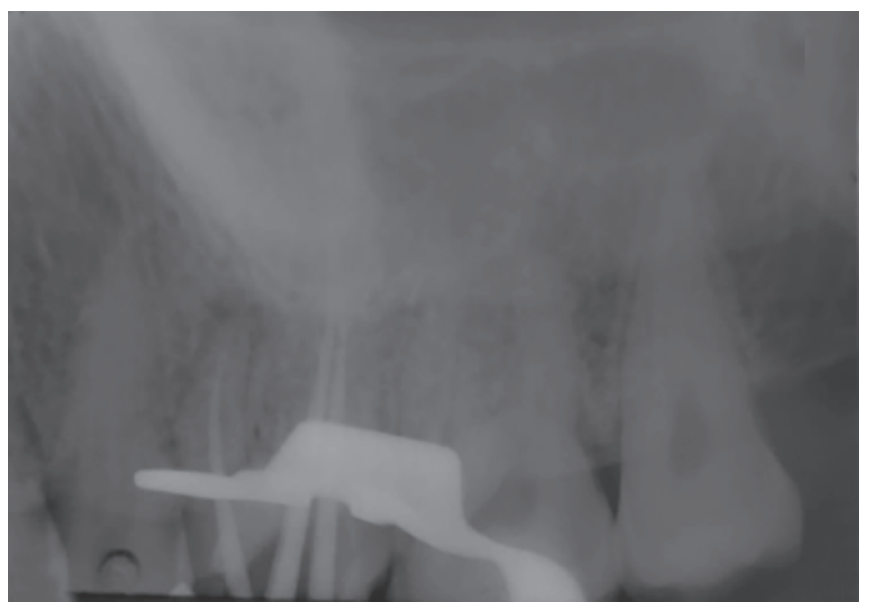

Fig. 15: Master cone

dent Apex(al) as an intracanal medicament and closed dressing was placed (Cavit G, 3M ESPE, Germany).

One week later, the patient was recalled. The patient was asymptomatic, and the medicament was removed with saline irrigation, the root canal was dried using paper point. Master cone fit was confirmed (Fig. 15). Root canals were obturated with guttapercha (25-4 and 20-6\%) along with AH Plus resin-based root canal sealer (Dentsply Maillefer) followed by composite (filtek Z350 XT, $3 \mathrm{M}$ ESPE, Germany) as post root canal filling material and coronal preparation was done and the impression was taken. After 2 days, PFM coronal prosthesis was delivered (Fig. 16).

\section{Discussion}

This case report outlines the basis of non-surgical endodontic management of a maxillary first molar with an anatomical variation in palatal root having two canals with Vertucci-type IV and Weine-type III root canal configuration confirmed with the help of CBCT.

In the present case, the conventional triangular access was modified into a trapezoidal form to improve access to the additional canals. Usually, the reading of a radiograph along with a careful inspection of the pulp chamber floor by probing and proper 
visualization enables the operator to locate and identify the root canal orifice. The use of endodontic explorer, surgical loupes, and electronic apex locator was crucial both for the detection and management of the two canals in the palatal root of maxillary molars. The clinician should thoroughly examine the pulpal floor and radiographs for the possibility of additional canals. ${ }^{4}$

The frequency of a maxillary first molar with two roots or two palatal canals is very low, 3.9 and $1 \%$ respectively. Properly designed and prepared access cavities to help the clinician diagnose and negotiate the root canal anatomy. ${ }^{2}$

Different methods should be used besides normal procedural protocols; evaluation of multiple radiographs at different angulation, white line test, red line test, piezoelectric ultrasonic device, troughing, champagne bubble test with sodium hypochlorite, nontoxic dye like $1 \%$ methylene blue, and dental operating microscopes (DOM) can be very helpful to locate extracanals. ${ }^{5}$

Proper identification of the canals and further endodontic management are essential for successful endodontic treatment. When there is only one canal, it is usually located rather easily in the center of the access preparation. If only one orifice is found and it is not in the center of the tooth, another canal is probably present and the operator should search for it on the opposite side. The relationship of the two canal orifices to each other is also significant. The closer the orifices are to each other, the greater are the chances that the two canals join at some point within the body of the root. ${ }^{2}$

Radiographic examination is an essential component for the management of endodontic problems. The amount of information gained from conventional radiographs and digitally captured periapical radiographs is limited by the fact that the threedimensional anatomy of the area being radiographed is compressed into a two-dimensional image. Cone-beam computed tomography permits a more accurate diagnosis of the aberrant root canals with accurate identifications and measurements by slicing of images in multiple planes which otherwise may not be easily identifiable with conventional radiographs even if taken at different angles. ${ }^{6}$

\section{Conclusion}

Anatomical variations in root canal morphology especially in molars is a challenge for endodontic therapy. It is important to consider these variations during root canal therapy of maxillary molars to prevent accidents and ensure a successful long-term outcome. We also accentuate the role of $\mathrm{CBCT}$ as an objective analytical tool to ascertain root canal morphology in unusual cases.

\section{References}

1. Hussain S, Mala K. Maxillary first molar with two palatal canals: a rare case report. Indian J Pub Health Res Develop 2018;9(1):176-180.

2. Biz Yeganeh LA, Adel M, Vahedi R, et al. Endodontic management of a maxillary first molar with two palatal canals and a single buccal canal: a case report. Case Rep Dent 2012;2012:1-4. DOI: 10.1155/2012/389387.

3. Filho B, Zaittar S, Haragushiku GA. Analysis of the internal anatomy of maxillary first molars by using different methods. J Endod 2009;35(3):337-342.

4. Bamboo J, Kumar M, Chandra S. Endodontic management of maxillary first molar with two palatal canals aided with CBCT: a case report. Compendium 2017;38(4):e1-e4.

5. Asghari V, Rahimi S. Treatment of maxillary first molar with 2 palatal canal. Iran Endod J 2012;10(4):287-289.

6. Stone LH, Stroner WF. Maxillary molars demonstrating more than one palatal root canal. Oral Surg, Oral Med, Oral Pathol 1981;51(6):649652. 